\title{
Similaridade acústica entre populações disjuntas de Formicarius colma Boddaert,1783 (Aves: Formicariidae)
}

\author{
Acoustic similarity among disjunct populations of \\ Formicarius colma Boddaert, 1783 (Aves: Formicariidae) \\ Mônica da Costa Lima', Erich de Freitas Mariano"
}

\section{Resumo}

O isolamento causado pela fragmentação do hábitat pode promover divergências morfológicas e comportamentais, mesmo em aves Suboscines. Formicarius colma é uma espécie de origem miocênica e que atualmente apresenta uma distribuição disjunta entre as florestas amazônica e atlântica e subdivide-se em quatro subespécies. Para avaliar se existem diferenças significativas entre o canto destas subespécies foram analisadas 73 gravações e utilizados 16 parâmetros bioacústicos. Para analisar a similaridade acústica foi utilizada a análise de componentes principais (PCA) e foram feitos teste T e ANOVA. O canto de F. colma consiste em um trinado na faixa de 2.4 a $3.5 \mathrm{kHz}$, com frequência dominante de $2,48 \mathrm{kHz}$ e duração média de $2,8 \mathrm{~s}$. A análise de agrupamento não aponta diferenças estatísticas entre as subespécies, mesmo entre as populações disjuntas. Contudo, é possível observar uma tendência de separação entre as populações amazônica e atlântica. Esta similaridade acústica pode estar relacionada a baixa plasticidade sonora encontrada nos Suboscines e as pressões que o meio exerce sob as populações não estão sendo suficientes para selecionar modulações distintas no canto das subespécies. Portanto, mesmo após um grande tempo de separação, não há diferenciação vocal significativa entre as populações estudadas.

Palavras-chave: WarbleR; Bioacústica; Biogeografia

\section{Abstract}

The isolation caused by habitat fragmentation may promote morphological and behavioral divergences, even in Suboscine birds. Formicarius colma is a Miocene specie, which has today a disjunct distribution among atlantic and amazonic forest and four subspecies. To evaluate significant differences among their subspecies vocalizations we analyze 73 records with 16 bioacoustic parameters. To analyze acoustic similarity we performed a Principal Component Analysis (PCA), t tests and ANOVA. The F. colma vocalization is a trill, ranging from 2.4 to $3.5 \mathrm{kHz}$, with dominant frequency of $2.48 \mathrm{kHz}$ and mean duration of $2,8 \mathrm{~s}$. The grouping analysis not shows statistics differences among F. colma subspecies vocalizations, even among disjunct populations. Howsoever, we can observe a tendency of segregation among amazonic and atlantic populations. This acoustic similarity may be relate to the low vocal plasticity founded in Suboscine and environmental pressure over these populations are not been adequate to select distinct modulations on subspecies song. Therefore, even a great separation time there are no significant vocal differences among studied population.

Keywords: WrableR; Bioacoustics; Biogeography 


\section{Introdução}

Aves Suboscines, na qual estão inseridos os Formicariidae, tendem a manter um padrão vocal sem muitas variações, mesmo com ampla distribuição geográfica (KROODSMA, 1984; DIAS, 2009). Entretanto, a fragmentação de populações biológicas tende a proporcionar divergências genotípicas, fenotípicas e comportamentais entre os membros segregados (IPPI, 2011; REPENNING, 2017). Uma dessas possíveis diferenciações estaria associada aos mecanismos de comunicação sonora e sua principal causa estaria relacionada às adaptações locais e ao acúmulo aleatório de mutações culturais (LYNCH, 1996; PODOS \& WARREN, 2007). A fragmentação de origem antropogênica, a colonização de ambientes isolados, distribuição anelar ou disjunções causadas por eventos de retração florestal podem ativar os mecanismos de segregação sonora (MORTON, 1975; NAUGLER \& SMITH, 1991; KROODSMA ET AL., 1999; BAKER, 1975; BAPTISTA \& KING, 1980; HARBISON ET AL., 1999; CICERO \& BRENOWITZ-FREDERICKS, 2000). Contudo, as relações entre a variação geográfica no canto e a fragmentação do hábitat muitas vezes não correspondem com as descontinuidades no hábitat, sendo muitas delas variações clinais (DALISO ET AL., 2015, RAMSAY \& OTTER, 2015).

Formicariidae iniciou seu processo de diversificação ainda no Paleogeno (cerca de 30 mya) e durante o Mioceno superior (cerca de 6,5 mya) as espécies de Formicarius modernas provavelmente diversificaram (SELVATTI ET AL., 2015; JETZ ET AL., 2012; CLARAMUNT, 2010). Nesse período a paisagem ao longo do Neotrópico era bem diferente do que vemos hoje (BATALHA-FILHO ET. AL., 2012; BATALHA-FILHO ET. AL., 2013; BATALHA-FILHO \& MYIAKY, 2016; CARVALHO ET AL., 2017). Palinofósseis de espécies de florestas úmidas e modelos de paleodistribuição sugerem uma conexão entre as formações de florestas úmidas na segunda metade do Mioceno superior (ORTIZ-JAUREGUIZAR \& CLADERA, 2006; POUND ET AL., 2012). Um modelo oriundo de uma matriz com dados de datação filogenética e distribuição de passeriformes florestais da América do Sul corrobora a ligação miocênica entre as florestas úmidas e apresenta um segundo evento de conexão entre estas duas formações florestais durante o Pleistoceno (BATALHA-FILHO ET AL., 2013). Esses eventos de flutuações florestais promoveram inúmeros isolamentos alopáticos entre as populações de aves, permitindo processos de diversificação e especiação e/ou a manutenção de distribuição disjuntas, como a que observamos em Formicarius colma.

Formicarius colma Boddaert, 1783, é um Formicariidae que persegue formigas de correição em florestas úmidas no Neotrópico. Atualmente são reconhecidas quatro subespécies de pouca variação morfológica, distribuídas ao norte do rio Amazonas (F. c. colma), ao sul do rio Amazonas e a leste do rio Madeira ( $F$. c. amazonicus), ao sul do rio Amazonas e a oeste do rio Madeira (F. c. nigrifrons) e ao longo da Floresta
Atlântica (F. c. ruficeps) (PIACENTINI ET AL., 2015; KRABBE \& SCHULENBERG, 2018).

De forma geral, o canto de $F$. colma dura cerca de cinco segundos, com um ritmo de 14 notas por segundo, iniciando de forma descendente e depois aumentando a tonalidade. Não são descritas grandes variações de canto entre as subespécies, porém em populações de F. c. ruficeps na Floresta atlântica da região sudeste do Brasil, foi observado que não há essa variação na tonalidade. Nessa população, as chamadas incluem um "pier" simples e claro, e um "psee-eh" como aquele de pintos doméstico, ambos muito diferentes das chamadas de outros membros do Formicarius (KRABBE \& SCHULENBERG, 2018).

Com base na disjunção de sua distribuição e nas diferenças do canto relatadas, nós buscamos avaliar se essas diferenças são significativas entre as subespécies de F. colma.

\section{Materiais e métodos}

Foram analisadas 73 gravações de F. colma (Figura 1) obtidas a partir do banco de dados do Xeno-canto (http:// www.xeno-canto.org). Os arquivos sonoros foram baixados com a função querxc do pacote warbleR (ARAYA-SALAS \& SMITH-VIDAURRE 2016) utilizando o software R (https://www.r-project.org). Todas as gravações analisadas correspondem as classificadas com qualidade A e B e vocalizações do tipo canto, tendo sido convertidas do formato original em MP3 para o formato WAVE.

As detecções dos cantos com a melhor qualidade dentro de cada gravação foram realizadas de forma manual usando o programa Audacity (https://www.audacityteam. org). Os parâmetros bioacústicos foram aferidos com ferramenta semi-automatizada do pacote warbleR, totalizando 28 parâmetros (APÊNDICE 1).

O primeiro passo nas análises dos dados foi reduzir o número de variáveis que caracterizam os cantos por meio de uma Análise de Componentes Principais (PCA) e a partir de uma análise de correlação, tendo sido excluídas ao menos uma dentre variáveis que apresentassem correlação igual ou superior a 0,8. A adequação da PCA foi avaliada a partir das métricas de Kaiser-Meyer-Olkin e do teste de esfericidade de Bartlett. Ambos os testes foram realizados com suas respectivas funções no pacote psych (REVELLE, 2018). A normalidade das variáveis foi verificada através do teste de Shapiro-Wilk $(p<0,001)$, e aquelas que não se adequavam aos pressupostas da normalidade foram logaritimizadas.

Para avaliar a similaridade acústica entre as populações foi realizado uma PCA com os registros sonoros obtidos. Os escores dos componentes gerados foram associados aos sons de cada localidade. A similaridade entre as populações da Floresta Atlântica e Amazônica foram comparadas com o uso dos autovalores obtidos no PCA para cada registro e comparadas pelo teste $t$. A similaridade entre as subespécies avaliadas foi testada usando uma ANOVA. Todas as análises foram realizadas com o software R 3.4.3 ( https://www.r-project.org). 
Figura 1 - Área de distribuição das subespécies de Formicarius colma e localidades dos registros do canto com qualidade A e B disponíveis no Xenocanto

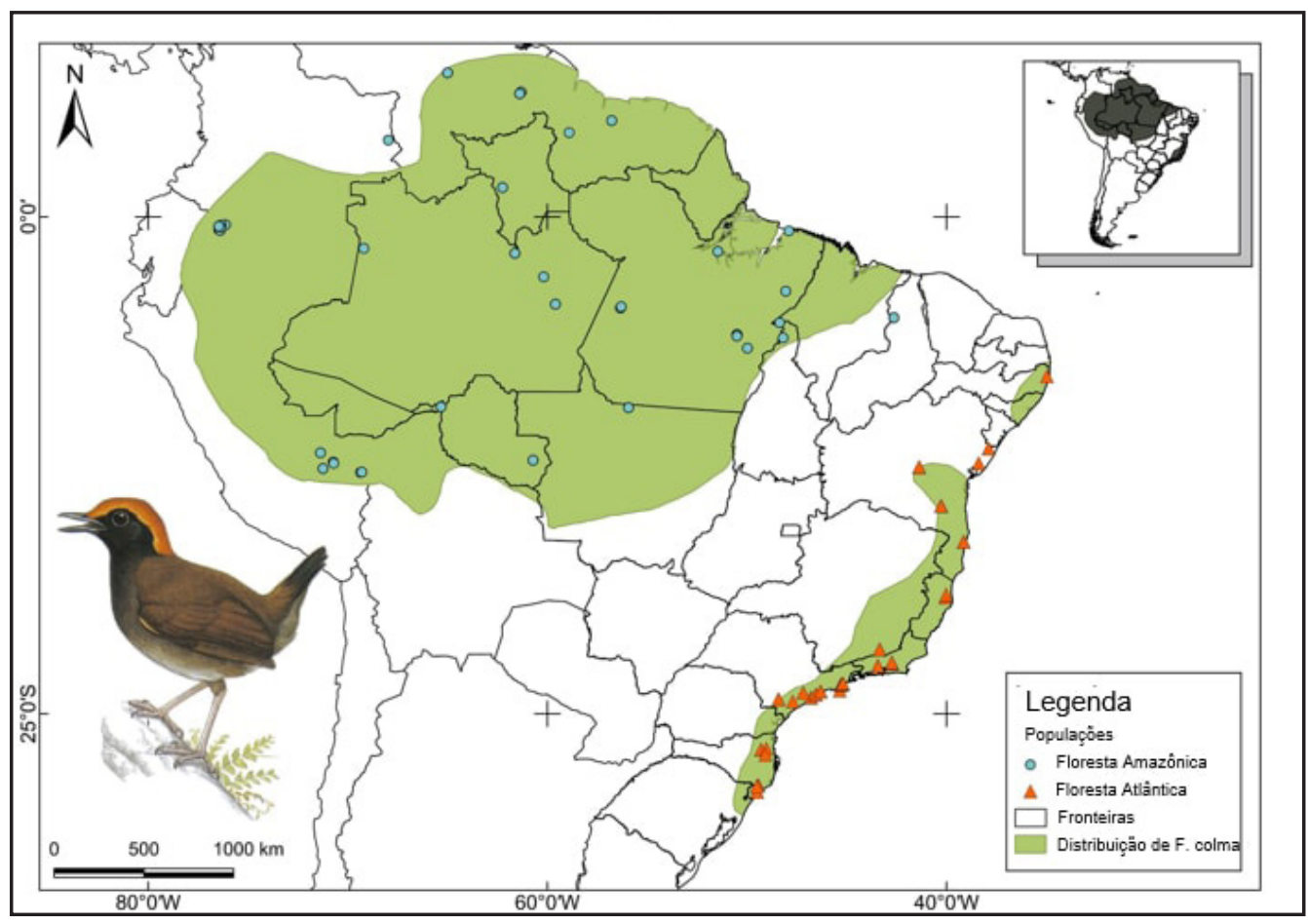

\section{Resultados}

O canto de F. colma consiste em um trinado composto por notas seriadas, concentrado em uma banda de frequência entre 2.4 a $3.5 \mathrm{kHz}$, com frequência dominante média de $2.48 \mathrm{kHz}$, e duração média de 2.84 segundos (Figura 2).
Foram selecionadas 16 variáveis dentre as 28 aferidas pela função semi-automatizada do warbleR. O resultado do teste $t$ indica que não há diferenças estatísticas entre o canto das populações estudadas $(t=-1.4898$, $\mathrm{df}=43.21, p=0,2925)$ e a ANOVA também não indica diferenças significativas as subespécies $(\mathrm{F}=0.943 . p=$ 0.425). Contudo, na ordenação obtida através da PCA é

Figura 2- Espectograma do canto de Formicarius colma ( $F F T=2000$ ) obtido com a função spec do pacote seewave no R 3.4.3

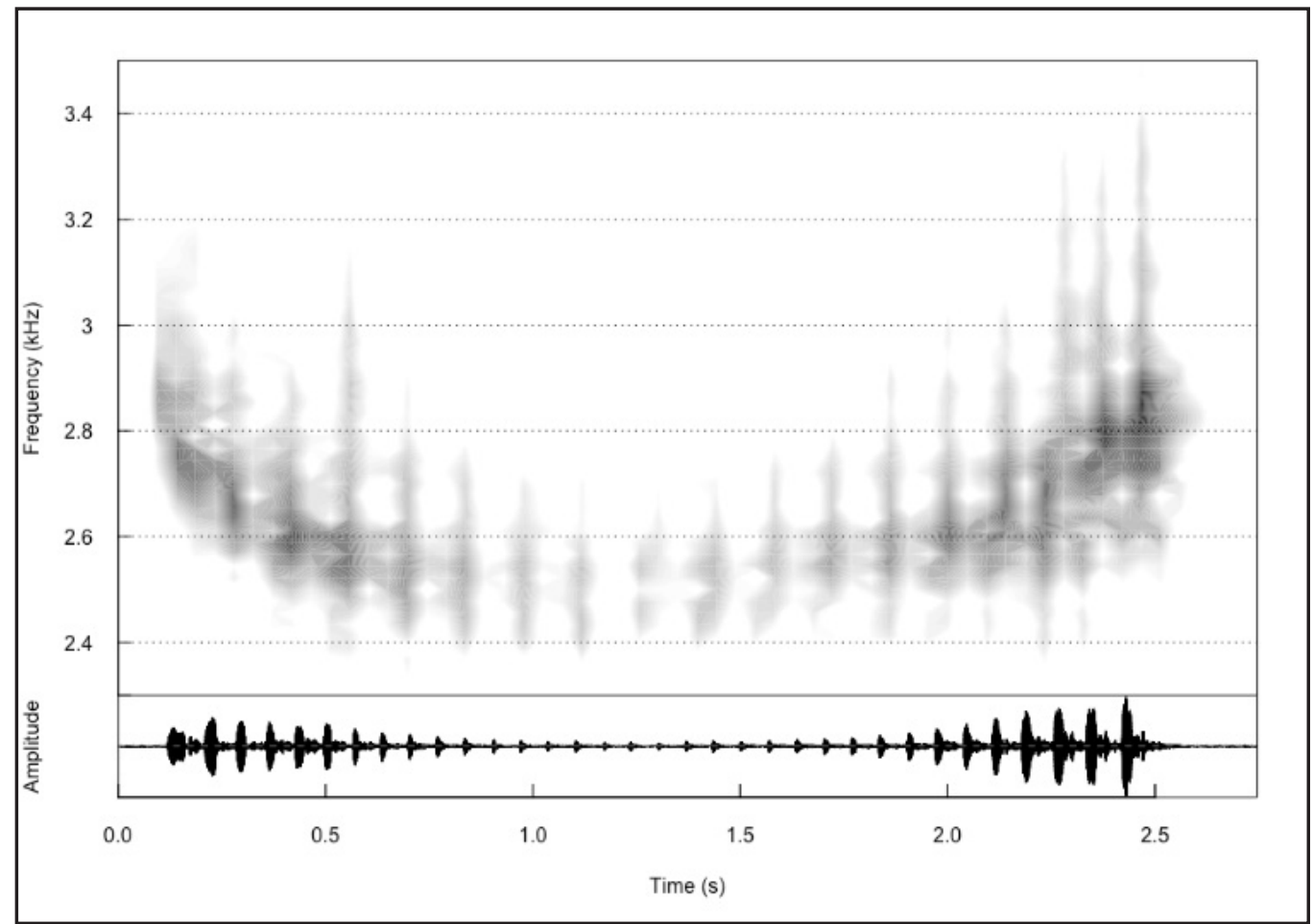


Figura 3 - Resultado do PCA: a) similaridade no canto de Formicarius colma de populações disjuntas b) agrupamentos por subespécies
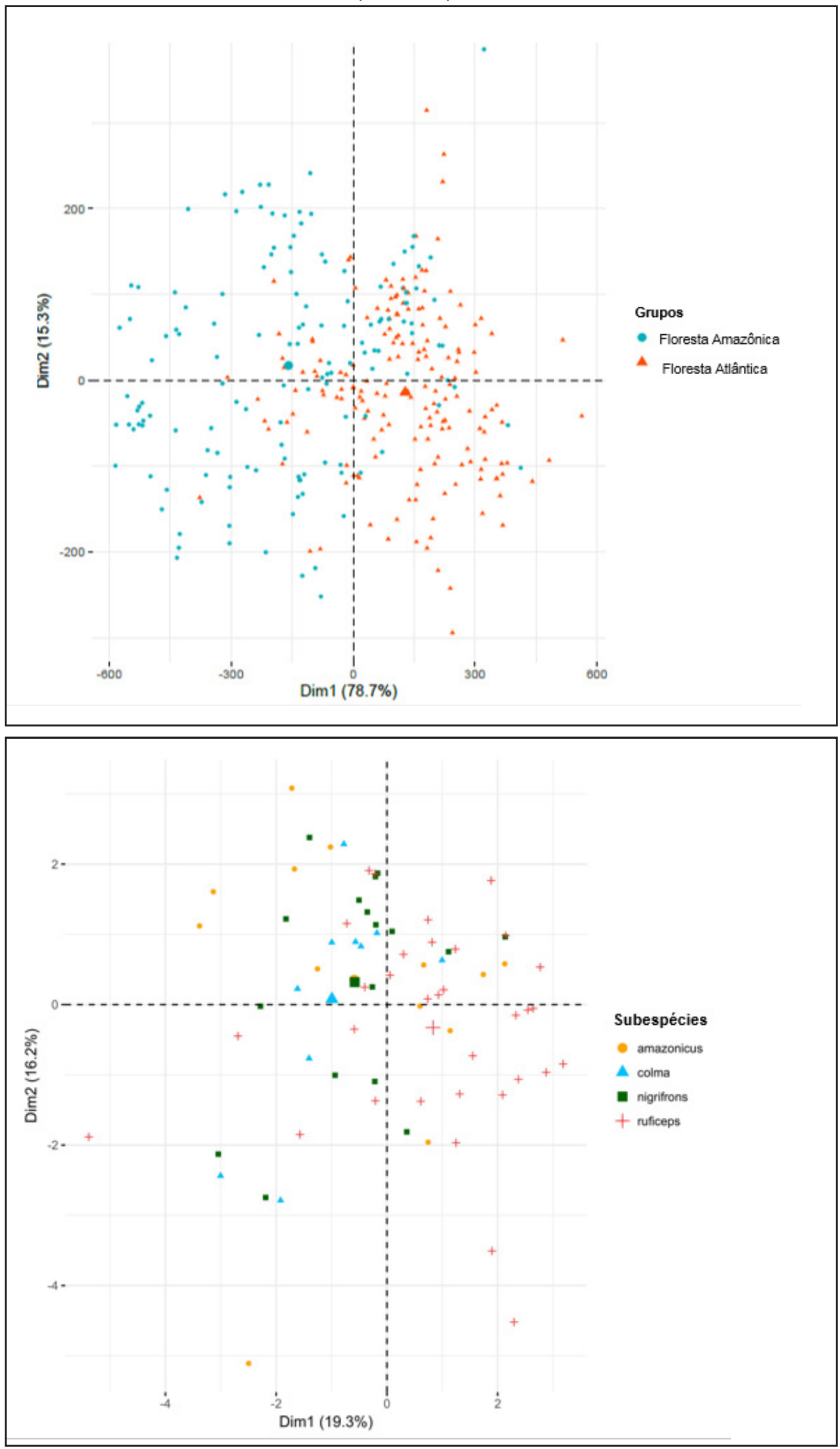
possível observar uma tendência de separação entre as populações amazônicas e da Floresta Atlântica (Figura 3a). A figura $3 \mathrm{~b}$ apresenta as relações de agrupamento entre as subespécies.

\section{Discussão}

Apesar do tempo de separação das formações de florestas úmidas (Floresta Amazônica e Floresta Atlântica) ser bastante antigo (SELVATTI ET AL., 2015; JETZ ET AL., 2012; CLARAMUNT, 2010), este não foi suficiente para permitir mudanças significativas no canto de $F$. colma, como apontam os testes estatísticos. Apesar dessa similaridade estatística, a distribuição das vocalizações na PCA sugere uma tendência à separação entre as disjunções. Essa sutil diferença pode ser observada em registros de naturalistas, como os observadores de aves. Tais registros apontam uma leve horizontalidade (menor amplitude de frequência) no trinado da subespécie da região Sudeste do Brasil (F. c. ruficeps) (KRABBE \& SCHULENBERG, 2018).

Disjunções, como a observada entre as florestas Amazônica e Atlântica, atuam como ecossistemas isolados, no que diz respeito a geração de variações geográficas pela vicariância. O que se espera em estudos que trabalham com populações separadas é que áreas isoladas entre si exibam vocalizações com características distintas e que haja pouca ou nenhuma variação no canto entre os indivíduos dentro de uma área contínua (JANES, RYKER E RYAN, 2017).

Muitos dos trabalhos sobre variações geográficas nos cantos de aves são feitos com Oscines (CATALANO, 2015). Todavia, o presente estudo, realizado com um Suboscine (Formicaridae, F. colma), nos fornece a oportunidade de comparar sinais acústicos entre grupos disjuntos (Amazônico e Atlântico) e sem as tendências causadas pelo processo de aprendizagem do canto e evolução cultural que ocorre nos Oscines.

Suboscines constituem, dentro das aves neotropicais um grupo caracterizado pela presença de uma siringe mais simples que os Oscines, repertório reduzido e canto fortemente estereotipado ao ponto de filhotes criados longe dos pais terem mesmo padrão vocal (AMES, 1972; KROODSMA, 1984; SIBLEY E MONROE, 1990). O surgimento de uma pequena variação do canto poderia limitar a reprodução e posteriormente poderia gerar uma pressão seletiva na formação de parceiros (SLABBEKOORN E SMITH, 2002). Trabalhos como os de Ippi et al. (2001) mostram também que, apesar do canto se desenvolver de forma inata, pequenas variações, mesmo que não significantes, surgem neste grupo. Vale ressaltar que, diferenças vocais em Suboscines não significa que houve aprendizado em algum estágio da vida da ave, mas talvez um surgimento de diferenças físicas, limitações no desenvolvimento ou diferenças genéticas (GIL E GAHR, 2002).

A emissão de um sinal sonoro está intimamente relacionada com as pressões que as interferências ambientais podem causar (MENDONÇA, 2009). Deste modo, formas melhor adaptadas as mudanças ambientais podem ser selecionadas e gerarem pequenas diferenças no contexto populacional (PODOS E WARREN, 2007). A fragmentação do hábitat pode proporcionar o surgimento de novas pressões ambientais sobre o canto das espécies.

Apesar da tentativa de relacionar a fragmentação do hábitat com as vocalizações ter se mostrado pouco clara (JANES, RYKER E RYAN, 2017), a hipótese da Competição Acústica (NAUGLER E RATCLIFFE, 1994) propõe que a diversidade ou complexidade sonora irá aumentar com a diminuição do fragmento florestal. Desta forma, o declínio na riqueza de espécies causado pela fragmentação disponibiliza nichos no espaço acústico anteriormente utilizados, proporcionando um aumento na riqueza ou complexidade sonora das populações (HART ET AL., 2018).

\section{Conclusões}

Mesmo que o tempo de separação entre as populações de F. colma seja antigo (Mioceno ou Pleistoceno), esse tempo não foi suficiente para causar alterações no canto de forma significativa. Vale ressaltar que existe uma tendência de separação entre os cantos da Floresta Amazônica e da Floresta Atlântica. Este padrão de diferenciação pode estar relacionado a alta taxa de fragmentação das populações da Floresta Atlântica, a qual pode permitir um aumento na complexidade ou variação no padrão vocal. Sugere-se que estudos que testem a influência da fragmentação sobre o canto de espécies Suboscines sejam realizados, bem como estudos genéticos e morfológicos afim de testar a similaridade e obtermos um melhor entendimento dos processos que levaram as disjunções de distribuição das aves neotropicais.

\section{Referências}

AMES PL. The morphology of the syrinx in passerine birds. Peabory Mus. Nat. Hist. 1971; 37:1-194.

ARAYA-SALAS M, SMITH-VIDAURRE G. WarbleR: an r package to streamline analysis of animal acoustic signals. Methods Ecol Evol. 2016;doi:10.1111/2041-210X.12624.

BATALHA-FILHO H, CABBANE GS, MIYAKI CY. Phylogeography of an Atlantic forest passerine reveals demographic stability through the last glacial maximum. 2012;3:892-902.

BATALHA-FILHO H, MIYAKI CY. Late pleistocene divergence and postglacial expansion in the Brazilian Atlantic forest: multilocus phylogeography of Rhopias gularis (Aves: Passeriformes). Journal of Zoological Systematics and Evolutionary Research. 2016;54:137-147. 
BATALHA-FILHO H.; FJEIDSA J, FABRE PH, MIYAKI CY. Connections between the Atlantic and the Amazonian forest avifaunas represent distinct historical events. J. Ornhitol. 2013;154: 41-50.

BAKER HG. Concentrations in nectars from hummingbird flowers. Biotropica. 1975;7:37-41.

BAPTISTA LF, KING JR. Geographical variation in song and song dialects of montane White-crowned Sparrows. Condor.1980;82:267-284.

CARTALANO ALC. Variação do canto de thamnophilus doliatus (linnaeus, 1764) (aves: thamnophilidae) em diferentes regiões da américa latina [dissertation]. São Paulo: Instituto de Biociências de Botucatu/ UNESP;2015.56p.

CARVALHO CS, NASCIMENTO NFF, ARAÚJO HFP. Bird distributional patterns support biogeographical histories and are associated with bioclimatic units in the Atlantic Forest, Brasil. Zootaxa. 2017;2:223-242.

CICERO CC, BENOWITZ-FREDERICKS ZM. Song types and variation in insular populations of Lincoln's Sparrow (Melospiza linconii) and comparispons with others Melospiza. The Auk. 2000;117:52-64.

CLARAMUNT S.Discoering exeptional diversifications at continental scales: The case of the endemic families of neotropical subocine passerines. Evolution. 2010;64:20042019.

DALISO AC, JENSEN WE, PARKER TH. Divergence of vocal culture among isolated alpine habitats is inconsistent among three oscine species. J Ornithol. 2015;156: 165-178.

DIAS AFS. Comparação e descrição de parâmetros acústicos do canto da Volatinia jacarina (Aves: Emberezidae) no contexto de seleção sexual [dissertation]. Brasilia: Instituto de biologia/UNB; 2009. 70p.

GIL D, GAHR M. The honesty of bird song: Multiple contraints for multiple traits. Trends in ecology \& evolution. 2002;17:133-141.

HARBISON H, NELSON DA, HAHN TP. Long-Term persistence of song dialects in the mountain WhiteCrowned Sparrow. The condor. 1999;101:133-148.

HART PJ, GONZALEZ ES, TANIMOTO A, THOMPSON A, SPEETJENS T, HOPKINS M, et. Al. Birdsong characteristics are related to fragment size in a neotropical forest. Elsevier. 2018;137: 45-52.

IPPI S, VÁSQUEZ RA, DONGEN WFDV, LAZZONI I. Geographical variation in the vocalizations of the suboscine Thorn-tailed Rayadito Aphrastura spinicauda. 2011;153:789-805.
JANES SW, RYKER L, RYAN RM. Yellow-Rumped Warbles use two songs categories: Each male tends to use a unique song in each category in southern Oregon. Northwestern Naturalist. 2017; 98:1-7.

JETZ W, JOY JB, HARTMANN K, MOOERS AO. The global diversity of birds in space and time. Nature. 2012:491.

KRABBE, NK, SCHULENBERG, TS. Rufous-capped Antthrush (Formicarius colma). In: del Hoyo, J., Elliott, A., Sargatal, J., Christie, D.A. \& de Juana, E. (eds.). Handbook of the Birds of the World Alive. [Internet] 2018. [Cited 2018 march 20). Available from: https://www.hbw.com/ species/rufous-capped-antthrush-formicarius-colma.

KROODSMA DE. Song of the Aldern Flycatcher (Empidomax alnorum) and Willow Flycatcher (Empidonax traollii) are innate. The Auk; 1984;101: 13-24.

KROODSMA DE, SÁNCHEZ J, STEMPLE DW, GOODWIN E, SILVA ML, VIELLIARD J. Sedentary life style of neotropical sedge Wrens promotes song imitations. Anim. Behav. 1999;57: 855-863.

LYNCH M. Mutation accumulation in transfer RNAs: Molecular evidence for Muller's Ratchet in mitochondrial genomes. University of Oregon. 1996;13; 209-220.

MENDONÇA ET. A utilização de Herpsilochmus longirostris pelzeln, 1868 (Passeriformes: Thamnophilidae) como modelo de adaptação acústica. [dissertation]. Uberlândia: Universidade Federal de Uberlândia/UFU;2009.22p.

MORTON ES. Ecological sources of selection on avian sound. Jstor; 1975;109:17-34.

NAUGLER CT, RATCLIFFE L. Character Release in bird song: A test of the acoustic competition hyphotesis using American tree sparrows Spizzela arborea. Journal of avian biology. 1994;25:142-148.

NAUGLER CT, SMITH PC. Song similarity in an isolated population of Fox Sparrows (Passerella iliaca). Condor. 1991;93:1001-1003.

ORTIZ-JAUREGUIZAR E，CLADERA GA. Paleoenviromental evolution of Southern South America during the Cenozoic. Journal of arid Enviroments. 2006; 66:498-532.

PIACENTINI VQ, ALEIXO A, AGNE CE, MAURICIO GN, PACHECO JF, BRAVO GA, BRITO GRR, NAKA LN, OLMOS F, POSSO S, SILVEIRA LF, BETINI GS, CARRANO E, FRANZ I, LEES AC, LIMA LM, PIOLI D, SCHUNCK F, AMARAL FR, BENCKE GA, COHNHAFT M, FIGUEIREDO LFA, STRAUBE FC \& CESARI E. 
Annotated checklist of the birds of Brazil by the Brazilian Ornithological Records Committee / Lista comentada das aves do Brasil pelo Comitê Brasileiro de Registros Ornitológicos. Revista Brasileira de Ornitologia. 2015; 23(2): 91-298.

PODOS MJ, HAYWOOD AM, SALZMANN U, RIDING JB. Global vegetation dynamics and latitudinal temperature gradients during the Mid to late Miocene (19.97-5.33 MA). Elsevier. 2012; 112:1-22.

PODOS JM, WARREN.PS. The evolution of geographic variation in birdsong. Advances in the study of behavior or Elsevier. 2007; 37:403-458.

RAMSEY SM, OTTER K. Geographic variation in Whitethroated Sparrow song may arise through cultural drift. Journal of Ornithology. 2015; 156:763-773.

R Core Team (2017). R: A language and environment for statistical computing. R Foundation for Statistical Computing, Vienna, Austria. URL https://www.R-project.org/.

REPENNING M. Variação geográfica em Sporophila (Aves: Thraupidae) com base em evidências fenotípicas, ecológicas e genéticas [thesis]. Rio Grande do Sul: Faculdade de biociências/PUCRS; 2017.228p.

REVELLE W. psych: Procedures for Personality and Psychological Research, Northwestern University, Evanston, Illinois, USA, 2018. https:/CRAN.R-project. org $/$ package $=$ psych Version $=1.8 .4$.

SIBLEY CG, MONROE BL.JR. Distribution and Taxonomy of Birds of the World. 1990. New Haven \& London: Yale University Press. 1990. .[Internet] Available from:: https:// yalebooks.yale.edu/book/9780300049695/distribution-andtaxonomy-birds-world

SLABBEKOORN H, SMITH TB. Bird song, ecology and speciation. Phil. Trans. R. Soc 2002;357:493-503.

SELVATTI AP, GONZAGA LP, RUSSO CAM. A paleogene origin for crown passerines and the diversification of the Oscines in the word. Elsevier. 2015;88:1-15. 